\title{
Surface Enhanced Raman Scattering on Silver sinusoidal nano-grating: Impact of Interactions of grating-coupled Surface Plasmon polaritons
}

\section{Zhaoyi Chen}

Peoples Liberation Army Engineering University

Ke Feng ( $\square$ handan50511@163.com )

Peoples Liberation Army Engineering University https://orcid.org/0000-0002-7592-6284

\section{Zhibin Chen}

63908 troops of the PLA

\section{Jinxing Shen}

Peoples Liberation Army Engineering University

\section{Huanliang Li}

Peoples Liberation Army Engineering University

\section{Research Article}

Keywords: surface plasmon polariton (SPP), Surface-enhanced Raman Scattering (SERS), sinusoidal nano-grating, odd modes, SPP coupling

Posted Date: August 10th, 2021

DOI: https://doi.org/10.21203/rs.3.rs-761365/v1

License: (c) (i) This work is licensed under a Creative Commons Attribution 4.0 International License. Read Full License 


\title{
Surface Enhanced Raman Scattering on Silver sinusoidal nano-grating: Impact of Interactions of grating-coupled Surface Plasmon polaritons
}

\author{
Zhaoyi Chen ${ }^{1}$, Ke Feng ${ }^{1,}$ *, Zhibin Chen ${ }^{2}$, Jinxing Shen ${ }^{1}$, Huanliang Li ${ }^{1}$ \\ 1. Peoples Liberation Army Engineering University, Nanjing, Jiangsu, China; \\ 2. 63908troops of the PLA, Shijiazhuang 050003, China; \\ * Corresponding author, E-mail: handan50511@163.com
}

\begin{abstract}
In this study, we reported a silver sinusoidal nanograting used in microchannels, forming $\mathrm{H}_{2} \mathrm{O} / \mathrm{Ag} / \mathrm{NOA}$ heterostructure, and studied the impact of interactions of grating-coupled surface Plasmon polaritons (SPPs) on Surface-enhanced Raman Scattering (SERS). FDTD simulations showed that when the refractive index of NOA is close to that of $\mathrm{H}_{2} \mathrm{O}$, there were two modes of odd coupling and even coupling between SPPs. Additionally, the thinner the Ag grating, the stronger the coupling, accompanied by the frequency shift of the two coupling modes. We also estimated the influence of refractive index of the surrounding medium on SPPs coupling by varying the dielectric of the upper and lower layer of Ag grating. Our experimental results were supported by FDTD calculations, which confirmed the importance of the interactions of grating-coupled SPPs in the design of SERS substrate.
\end{abstract}

Keywords : surface plasmon polariton (SPP), Surface-enhanced Raman Scattering (SERS), sinusoidal nanograting, odd modes, SPP coupling

\section{Introduction}

Nanostructures that support surface plasmon resonance (SPR) have been widely used for sensing applications through the use of different techniques, including SPR spectroscopy ${ }^{[1,2]}$, SPR imaging ${ }^{[3]}$ and surface-enhanced Raman scattering (SERS) spectroscopy ${ }^{[4-7]}$. SERS, particularly, allows for highly sensitive detection and specific identification of analytes, has been extensively employed in multiple applications including trace molecules detection ${ }^{[8,9]}$, biosensors $^{[10,11]}$, food security ${ }^{[12,13]}$, and environment safety ${ }^{[14,15]}$. Metallic nano-gratings ${ }^{[16,17]}$, in particular, experience large-area uniform electromagnetic enhancement $^{[18,19]}$, which increases the chances for analyte detection via SERS spectroscopy ${ }^{[19-21]}$. Their properties are connected to surface plasmon polariton (SPP), whose resonance wavelength depends on the geometrical parameters of the nanograting, the incident light and the surrounding medium ${ }^{[7]}$. 1D Metallic nanograting may enhance the electromagnetic field intensity at a metal-dielectric boundary near-surface region by $\sim 10$ times $^{[22]}$. However, the Raman spectroscopy is greatly affected by the surrounding medium when metallic nanograting is used to detect trace molecules in microchannels ${ }^{[23]}$. When the refractive index of the superstrate and substrate is close, the excitation of SPP takes place at the metal-air interface or the metal-glass interface leading to the interaction of different modes ${ }^{[24]}$.

In this work, we report on the optical study of coupling modes of SPPs supported by silver sinusoidal nanograting deposited on a Norland Optical Adhesive (NOA) coated glass substrate used in microchannels, forming a $\mathrm{H}_{2} \mathrm{O} / \mathrm{Ag} / \mathrm{NOA}$ heterostructure. Then we estimated the impact of interaction of grating-coupled SPPs on the SERS, by varying the thickness of $\mathrm{Ag}$ grating, the refractive index of the surrounding medium of the superstrate and substrate. They confirm the importance and the impact of interactions of gratingcoupled SPPs in the design of SERS substrates by considering the appropriate grating constant in accordance with FDTD simulations. 


\section{Model and Theoretical Analysis}

\subsection{Model}

The SERS structure of sinusoidal nanograting with period $\Lambda$, consists of 3 layers $\left(\mathrm{Ag}, \mathrm{NOA}, \mathrm{SiO}_{2}\right)$, as seen in Fig.1. The medium above the $\mathrm{Ag}$ layer is $\mathrm{H}_{2} \mathrm{O}$, which functions as a concentrator, effectively increasing the volumetric density of trace molecules. Fabrication of the sinusoidal nanograting can be performed using the rapid and high-throughput interferometric technique

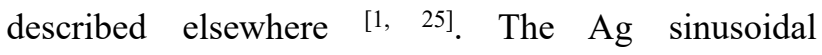
nanograting, to provide the metallic interface for the SPP excitation, has a large-area approach for target analyte detection, in contrast to established hot spot methods $^{[25]}$.

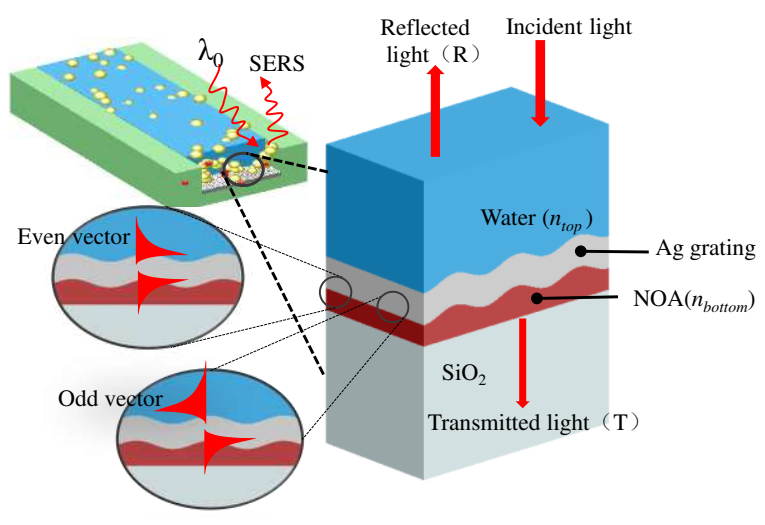

Fig. 1 Schematic diagram of the SERS substrate

The optical responses of the heterostructure were computed using FDTD simulations, as defined in Fig.2, by assuming that the grating volume extended uniformly in $\mathrm{Y}$ direction. So, periodic boundary conditions (PBCs) were used in the $\mathrm{X}$ - and Y-direction, while perfectly matched layers (PMLs) were used in the Z-direction. Normal incidence of light (plane waves) with transverse magnetic (TM) polarizations, were considered as excitations. The dispersive dielectric function of Ag was extracted from the experimental data from $\operatorname{Ref}^{[26]}$. The nondispersive refractive indices of $\mathrm{H}_{2} \mathrm{O}$ and glass $\left(\mathrm{SiO}_{2}\right)$ were considered as 1.3356 and 1.45 , respectively. Relatively fine grid spacings set with $1 \mathrm{~nm}$, and simulation time with $1000 \mathrm{fs}$ is applied for well converged results.

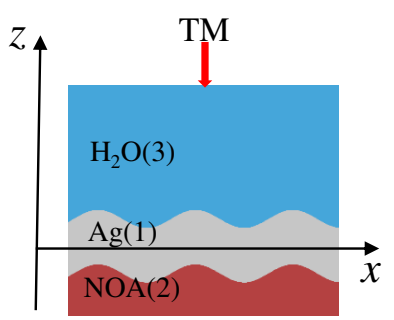

Fig. 2 Geometry of the $\mathrm{H}_{2} \mathrm{O} / \mathrm{Ag} / \mathrm{NOA}$ heterostructure

The periodicity of the Ag sinusoidal grating was set about 570nm calculated by using Eq.(2) for a laser excitation wavelength of $785 \mathrm{~nm}$, with the dispersive dielectric function of $\mathrm{Ag}$ from $\mathrm{Ref}^{[26]}$. The amplitude of sinusoidal grating was set about $20 \mathrm{~nm}$, with high electromagnetic enhancement ${ }^{[22]}$. The index of NOA varied from 1.33 to 1.7 in the wavelength range, corresponding to different dielectric.

\subsection{Theoretical Analysis}

The dispersion relation of SPP, propagating at a metal/dielectric interface, is defined as Formula (1).

$$
\beta=k_{0} \sqrt{\frac{\varepsilon_{m}(\omega) \times \varepsilon_{d}}{\varepsilon_{m}(\omega)+\varepsilon_{d}}}
$$

Where $\beta$ is the SPP wave vector, $k_{0}=\frac{\omega}{c}$ is the incident wave vector, $\omega$ and $c$ are the frequency and speed of the incident light, $\varepsilon_{m}(\omega)$ and $\varepsilon_{d}$ are metal and dielectric permittivity.

When an incident beam of light is focused on a metallic grating, the diffraction wave will be generated $^{[27]}$, which can directly couple to SPP if the momentum of the diffracted wave is matched with the grating surface ${ }^{[28]}$ via the grating equation ${ }^{[29]}$ :

$$
\beta=k_{0} n_{d} \sin \theta_{i} \pm m \frac{2 \pi}{\Lambda}
$$

Where $n_{d}$ is the refractive index of the medium, $\theta_{i}$ is the angle of the incident light with respect to the normal on the grating, $m$ is an integer and represents the grating diffractive order, $\frac{2 \pi}{\Lambda}=k_{g}$ is the reciprocal lattice vector of the grating, and $\Lambda$ is the period of the grating.

The SPP fields fall off exponentially along the direction/metal perpendicular to the grating surface ${ }^{[30]}$, with the evanescent decay length of the fields calculated 
by Formula (3):

$$
\delta=1 / \sqrt{\beta^{2}-\varepsilon k_{0}^{2}}
$$

In the $\mathrm{H}_{2} \mathrm{O} / \mathrm{Ag} / \mathrm{NOA}$ heterostructure (IMI), as seen in Fig.2, when the refractive index of NOA is close to $\mathrm{H}_{2} \mathrm{O}$ and the thickness of $\mathrm{Ag}$ grating is comparable to or smaller than the decay length of the interface mode, where $\delta=23.484 \mathrm{~nm}$ in the Ag at $\lambda_{0}=785 \mathrm{~nm}$, each single interface can sustain bound $\mathrm{SPP}^{[31]}$, and interactions between SPPs give rise to coupled $\operatorname{modes}^{[32]}$.

For TM modes light, according to Maxwell equations, the field components in $\mathrm{H}_{2} \mathrm{O}$ are

$$
\begin{aligned}
& H_{y}=A e^{i \beta x} e^{-k_{3} z} \\
& E_{x}=i A \frac{1}{\omega \varepsilon_{0} \varepsilon_{3}} k_{3} e^{i \beta x} e^{-k_{3} z} . \\
& E_{z}=-A \frac{\beta}{\omega \varepsilon_{0} \varepsilon_{3}} e^{i \beta x} e^{-k_{3} z}
\end{aligned}
$$

While in NOA, we get

$$
\begin{aligned}
& H_{y}=B e^{i \beta x} e^{k_{2} z} \\
& E_{x}=-i B \frac{1}{\omega \varepsilon_{0} \varepsilon_{2}} k_{2} e^{i \beta x} e^{k_{2} z} . \\
& E_{z}=-B \frac{\beta}{\omega \varepsilon_{0} \varepsilon_{2}} e^{i \beta x} e^{k_{2} z}
\end{aligned}
$$

Where $k_{i} \equiv k_{z, i}$ is the component of the wave vector perpendicular to the interface. In the Ag grating, the modes localized at the $\mathrm{H}_{2} \mathrm{O} / \mathrm{Ag}$ and $\mathrm{Ag} / \mathrm{NOA}$ interface couple, yielding

$$
\begin{aligned}
& H_{y}=C e^{i \beta x} e^{k_{1} z}+D e^{i \beta x} e^{-k_{1} z} \\
& E_{x}=-i C \frac{1}{\omega \varepsilon_{0} \varepsilon_{1}} k_{1} e^{i \beta x} e^{k_{1} z}+i D \frac{1}{\omega \varepsilon_{0} \varepsilon_{1}} k_{1} e^{i \beta x} e^{-k_{1} z} \\
& E_{z}=C \frac{\beta}{\omega \varepsilon_{0} \varepsilon_{1}} e^{i \beta x} e^{k_{1} z}+D \frac{\beta}{\omega \varepsilon_{0} \varepsilon_{1}} e^{i \beta x} e^{-k_{1} z}
\end{aligned}
$$

The requirement of continuity of $H_{y}$ and $E_{x}$ leads to equation (7) at the $\mathrm{H}_{2} \mathrm{O} / \mathrm{Ag}$ interface (suppose $z=h$ ) and equation (8) at the $\mathrm{Ag} / \mathrm{NOA}$ interface (suppose $z=-h)$.

$$
\begin{aligned}
& A e^{-k_{3} h}=C e^{k_{1} h}+D e^{-k_{1} h} \\
& A \frac{1}{\varepsilon_{3}} k_{3} e^{-k_{3} h}=-C \frac{1}{\varepsilon_{1}} k_{1} e^{k_{1} h}+D \frac{1}{\varepsilon_{1}} k_{1} e^{-k_{1} h}
\end{aligned}
$$

$$
\begin{aligned}
& B e^{-k_{2} h}=C e^{-k_{1} h}+D e^{k_{1} h} \\
& -B \frac{1}{\varepsilon_{3}} k_{3} e^{-k_{2} h}=-C \frac{1}{\varepsilon_{1}} k_{1} e^{-k_{1} h}+D \frac{1}{\varepsilon_{1}} k_{1} e^{k_{1} h}
\end{aligned}
$$

$H_{y}$ further has to fulfill the wave equation $\frac{\partial^{2} H_{y}}{\partial z^{2}}+\left(k_{0}^{2} \varepsilon-\beta^{2}\right) H_{y}=0$ in three distinct regions, via $k_{i}^{2}=\beta^{2}-k_{0}^{2} \varepsilon_{i}$, where $i=1,2,3$.

Solving the system of liner equations results in the relation of $k_{i}$ via

$$
e^{-4 k_{1} h}=\frac{k_{1} / \varepsilon_{1}+k_{2} / \varepsilon_{2}}{k_{1} / \varepsilon_{1}-k_{2} / \varepsilon_{2}} \times \frac{k_{1} / \varepsilon_{1}+k_{3} / \varepsilon_{3}}{k_{1} / \varepsilon_{1}-k_{3} / \varepsilon_{3}} .
$$

\section{Results and Discussion}

\subsection{Coupling Mode}

Firstly, we analyzed the coupling modes of SPPs of the $\mathrm{H}_{2} \mathrm{O} / \mathrm{Ag} / \mathrm{NOA}$ heterostructure with $40 \mathrm{~nm}$ thick $\mathrm{Ag}$ and NOA133. The index of NOA133 is 1.33 , leading to a better matching with $\mathrm{H}_{2} \mathrm{O}$ index and forming a symmetric environment. The variation of reflectivity, absorbance and transmittance of SERS with incident wavelength is shown in Fig. 3.

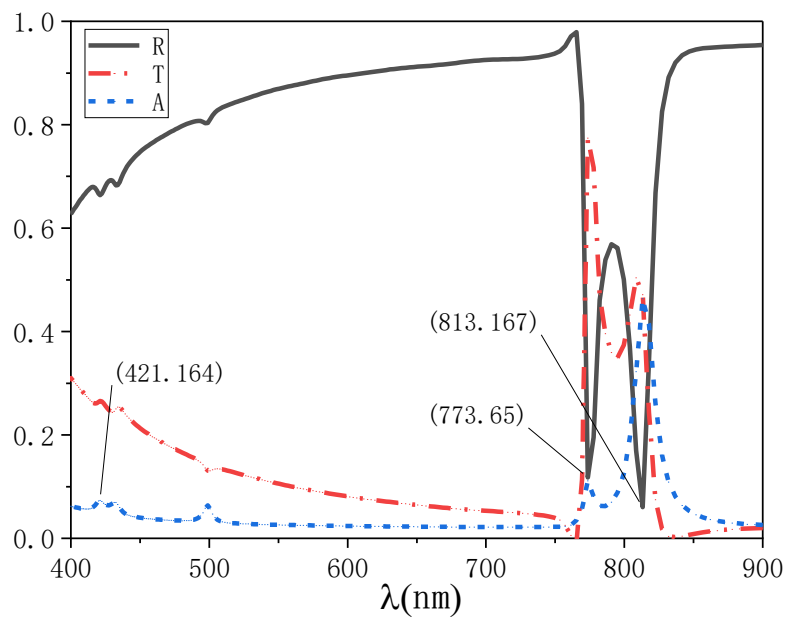

Fig. 3 Reflectance (R), transmittance (T), absorbance (A) of the SERS substrate as a function of wavelength of incident light $\left(\mathrm{H}_{2} \mathrm{O} / \mathrm{Ag} / \mathrm{NOA} 133\right.$ heterostructure, $\left.\mathrm{Ag}=40 \mathrm{~nm}\right)$

It can be seen that a strong coupling of SPPs occurs at $773 \mathrm{~nm}$ and $813 \mathrm{~nm}$ respectively, corresponding to the two resonance wavelengths, where the reflectance curve shows two sharp troughs, while two sharp peaks appear 
in the corresponding transmittance and absorbance curves. The electric field distributions of the SERS at resonance wavelength are shown in Figure 4.

A third maximum of intensity of absorbance near the $421 \mathrm{~nm}$ wavelength, with a much lower intensity, is observed, attributed to an order $(0, \pm 1)$ grating diffracted wave.
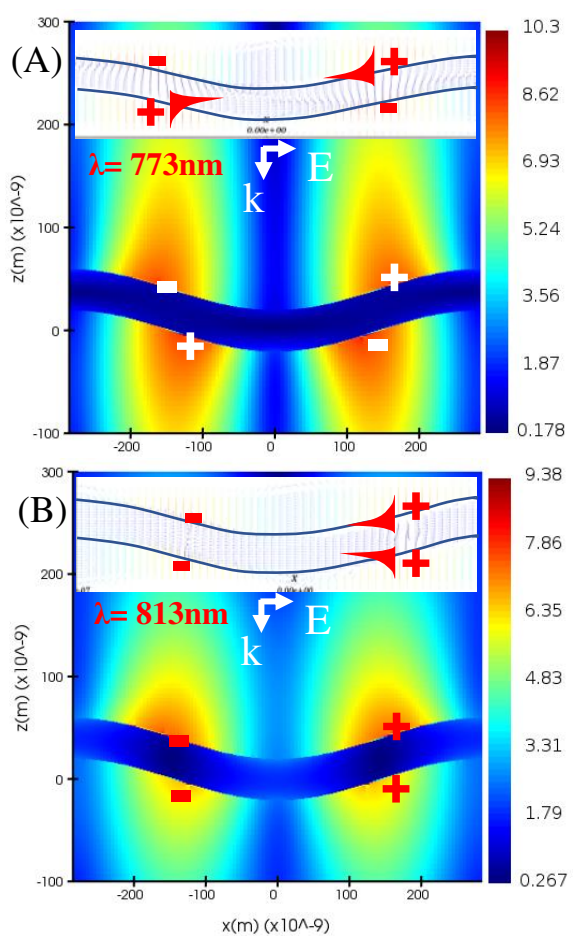

Fig. 4 Electric field distributions of the SERS substrate at (A) $\lambda=773 \mathrm{~nm}$ (B) $\lambda=813 \mathrm{~nm}$

When $\lambda=773 \mathrm{~nm}$, the interactions of SPPs result in odd vector $E_{x}(z)$ in the Ag grating. The SPP of the upper and lower interfaces propagates back along the $\mathrm{Ag}$ grating surface, and appears to repulse each other in the middle of the Ag layer, where appears extremely weak electric field. The $E_{x}(z)$ increases gradually from the middle of the Ag grating to the both interfaces. In this mode, the decay length of the evanescent field is larger in the dielectric, which forms a large range of electric field enhancement both in $\mathrm{H}_{2} \mathrm{O}$ and NOA.

When $\lambda=813 \mathrm{~nm}, E_{x}(z)$ in the $\mathrm{Ag}$ grating is even function, where $\mathrm{Ag}$ grating has strong bound on electromagnetic field, and the decay length of evanescent field is small in the dielectric.

The SPP couplings of both modes are strong in such structure, where the transmittance of SERS is large. In addition, the electric field of $\mathrm{Ag} / \mathrm{NOA}$ surface is almost equal to that of $\mathrm{H}_{2} \mathrm{O} / \mathrm{Ag}$ surface, with maximum of 4 electric intensity ( $E_{\max } / E_{0}$ ) of $\sim 10$ times.

\subsection{The effect of the thickness of Ag sinusoidal}

\section{grating}

According to the SPP dispersion equation (1), the theoretical SPP resonance wavelengths of $\mathrm{Ag} / \mathrm{NOA}$ and $\mathrm{H} 2 \mathrm{O} / \mathrm{Ag}$ interfaces are about $786 \mathrm{~nm}$ and $790 \mathrm{~nm}$, respectively. However, when $\mathrm{Ag}=40 \mathrm{~nm}$, the resonance frequencies shift due to the coupling of SPPs. In order to study the effect of thickness of Ag grating on SPPs coupling, FDTD simulations were carried out. The transmittance of SERS substrate varies with the thickness of Ag grating, as shown in Figure 5.

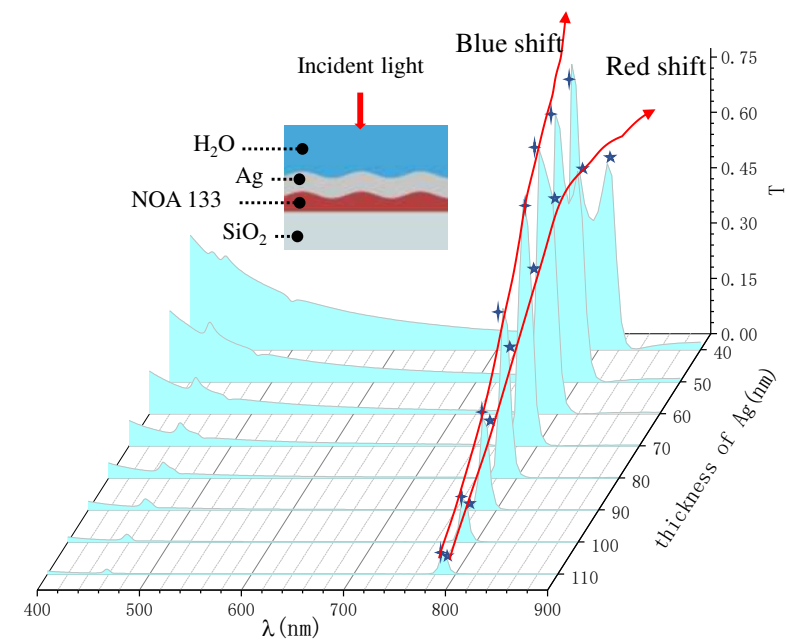

Fig. 5 Transmittance (T) of the SERS substrate as a function of thickness of Ag grating and wavelength of incident light $\left(\mathrm{H}_{2} \mathrm{O} / \mathrm{Ag} / \mathrm{NOA} 133\right.$ heterostructure, $\left.\mathrm{Ag}=40 \mathrm{~nm}\right)$

When the Ag grating is thick (such as $>100 \mathrm{~nm}$ ), the SERS substrate has two resonance wavelengths of $786 \mathrm{~nm}$ and $790 \mathrm{~nm}$, which are the same as the theoretical SPP resonance wavelength of $\mathrm{Ag} / \mathrm{NOA}$ and $\mathrm{H}_{2} \mathrm{O} / \mathrm{Ag}$ interfaces, respectively. Under this thickness, the transmittance is very small. As the thickness of $\mathrm{Ag}$ grating decreases, SPP coupling is enhanced, and the frequency of even modes of SPP coupling is redshifted, while that of odd modes is blue shifted, as shown in Figure 6. 


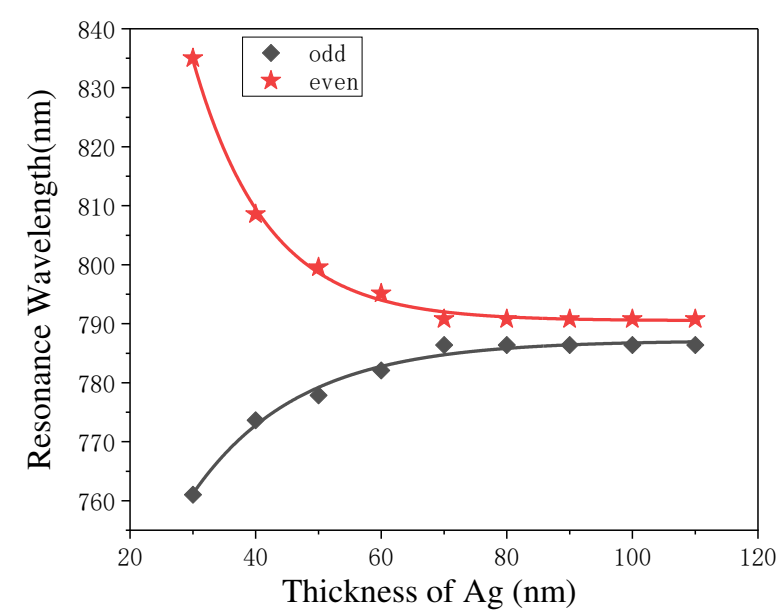

Fig. 6 The resonance wavelengths of the coupled modes as a function of thickness of Ag grating

For an incident wavelength $\lambda$, the transmittance increases with the decrease of $\mathrm{Ag}$ grating thickness. Because the skin depth of incident light in the $\mathrm{Ag}$ grating is small, the thicker the Ag grating, the less light penetration, the weaker the SPP excited at the interface $\mathrm{Ag} / \mathrm{NOA}$, which leading to the lower the coupling of SPPs. When the Ag grating is quite thick, even infinite $(h \rightarrow \infty)$, there is no electromagnetic field at the $\mathrm{Ag} / \mathrm{NOA}$ interface, let alone the coupling of SPPs, where the equation (9) can be approximated as two uncoupled SPP wave equations at their respective interfaces, as shown in Formula (10).

$$
\begin{aligned}
& k_{1} / \varepsilon_{1}=-k_{2} / \varepsilon_{2} \\
& k_{1} / \varepsilon_{1}=-k_{3} / \varepsilon_{3}
\end{aligned}
$$

For a certain thickness of $\mathrm{Ag}$ grating, the transmittance reaches the maximum when SPPs are strongly coupled. We also note that the transmittance is larger in the odd modes than that in even modes, mainly because the even modes have a stronger ability to bind the electromagnetic field.

The variation trend of reflectance is basically opposite to that of transmittance, as shown in Figure 7.

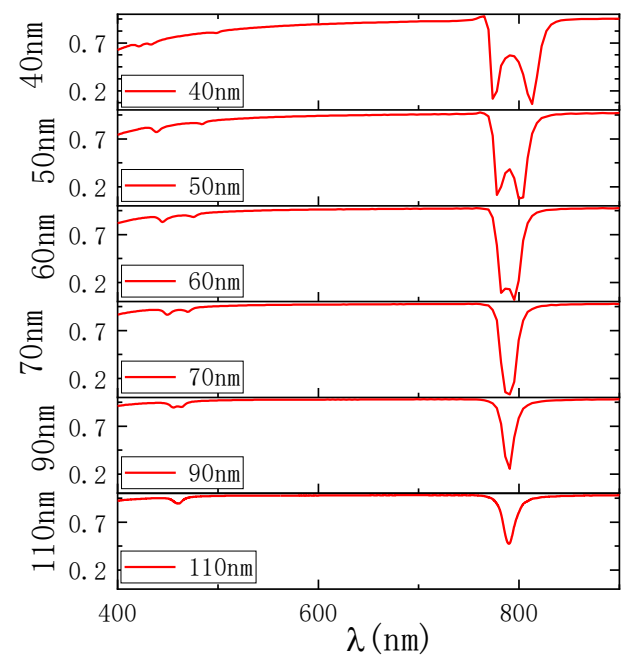

Fig. 7 Reflectance $(\mathrm{R})$ of the $\mathrm{H}_{2} \mathrm{O} / \mathrm{Ag} / \mathrm{NOA} 133$ system as a function of wavelength of incident light

The electric field distribution of the SERS substrate under resonance frequency are shown in Figure 8. When the $\mathrm{Ag}$ grating is relatively thin (such as $30 \mathrm{~nm}$ and $40 \mathrm{~nm}$ ), the coupling between SPPs is strong, where the maximum electric fields on both surfaces enhance nearly 10 times, in both odd and even modes. The decay length of the evanescent field in odd modes is larger, forming a larger range of electric field enhancement region in the medium.
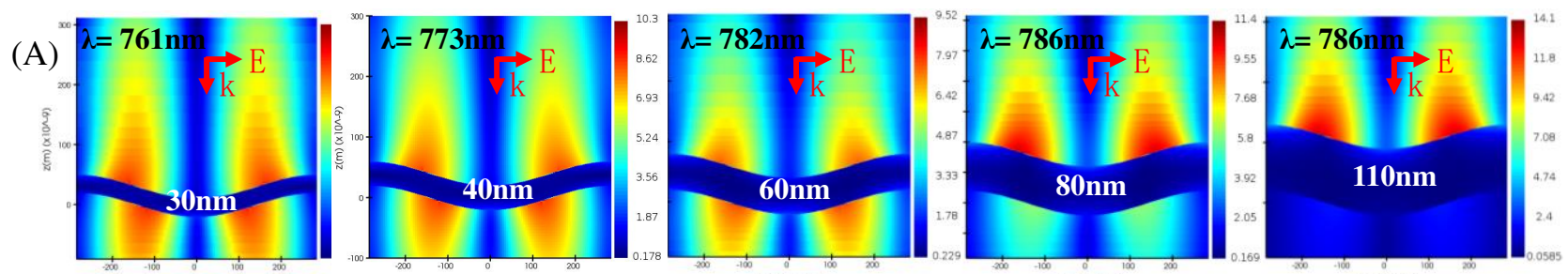

(B)
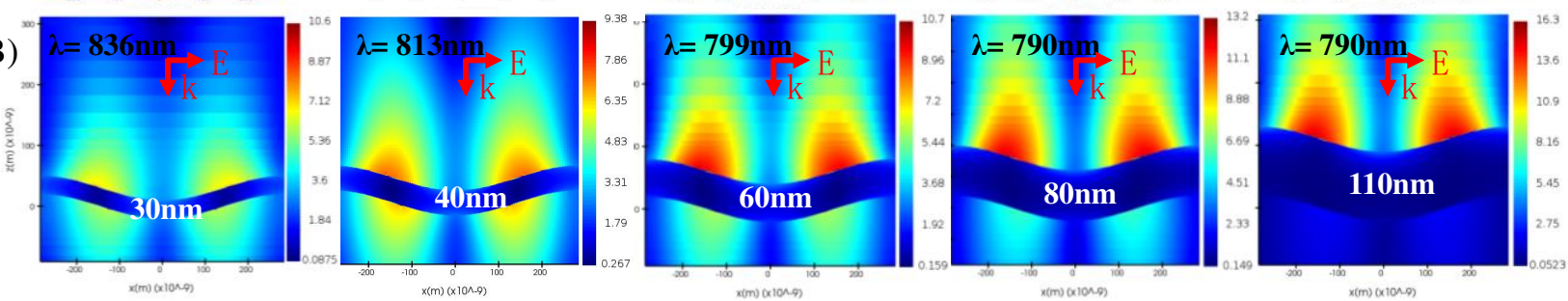

Fig. 8 Electric field distributions (A) $E_{x}(z)$ is odd function, (B) $E_{x}(z)$ is even function 
With the increase of Ag grating thickness, the SPPs and the electric fields on $\mathrm{Ag} / \mathrm{NOA}$ surface decrease, while that on $\mathrm{H}_{2} \mathrm{O} / \mathrm{Ag}$ surface increases gradually. It can be observed that the maximum electric field intensity occurs at the maximum $\mathrm{Ag}$ thickness (110nm). It can be said that the coupling of SPPs enhances the transmittance and the electric fields of $\mathrm{Ag} / \mathrm{NOA}$ surface, but at the same time reduces the electric fields on the surface of $\mathrm{H}_{2} \mathrm{O} / \mathrm{Ag}$, which is the strongest when the coupling is weak or even no coupling.

\subsection{The effect of the NOA}

In the following, we investigated the impact of the dielectric on the interaction of SPPs, as well as on the SERS. Different materials, such as NOA 142 (refractive index of 1.42), NOA 61 (refractive index of 1.56) and NOA 170 (refractive index of 1.7), were used for comparative experiments, and the influence of refractive index change on SERS was analyzed, as seen in Fig.9.

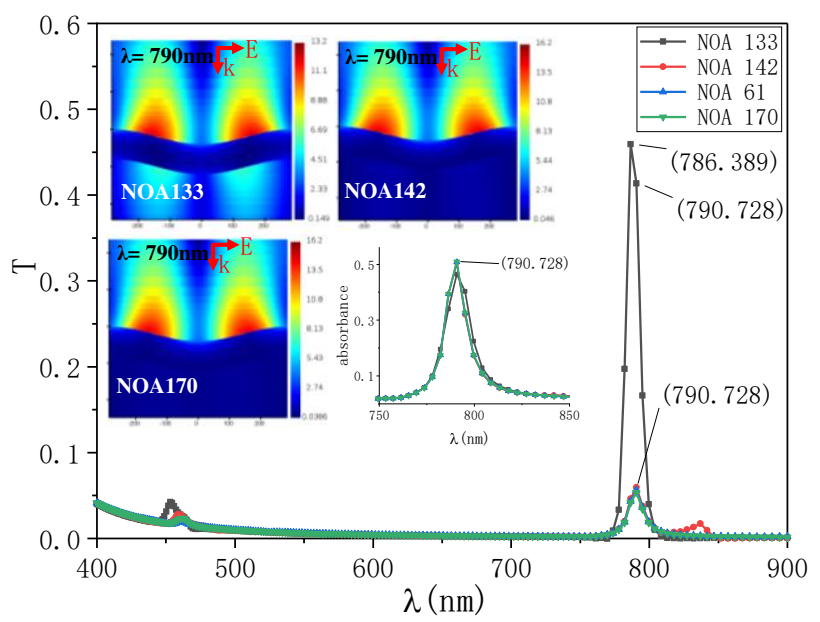

Fig. 9 SERS as a function of refractive index of NOA with $80 \mathrm{~nm} \mathrm{Ag}$

It should be noted that the coupling of SPPs does not cause the change of SPP resonance frequency on the $\mathrm{H}_{2} \mathrm{O} / \mathrm{Ag}$ surface when the thickness of $\mathrm{Ag}$ is more than $80 \mathrm{~nm}$, as illustrated in Figure 6. According to the SPP dispersion equation (1), the larger the refractive index of NOA dielectric, the longer the SPP resonance wavelength of Ag/NOA surface.

For absorbance, there is a good qualitative agreement for different NOA dielectric, and the maximum is obtained at $\lambda=790 \mathrm{~nm}$ On the contrary, the 6 transmittance curve is very different near SPP resonance wavelength. The maximum corresponds to NOA133 at $\lambda=786 \mathrm{~nm}$, since the refractive index of NOA133 and $\mathrm{H}_{2} \mathrm{O}$ is the closest, the coupling of SPPs leads to a sharp increase in transmission of SERS substrate under $786 \mathrm{~nm}$ incident light. That is to say in a symmetric environment, the SPPs of the two interfaces are expected to be symmetric, and thus with a higher coupling. However, the refractive indices of other NOA materials and $\mathrm{H}_{2} \mathrm{O}$ are quite different, which hinder the coupling of SPPs between the interfaces, resulting in extremely low electric field intensity in NOA. On the contrary, the electromagnetic field on the $\mathrm{H}_{2} \mathrm{O} / \mathrm{Ag}$ surface is stronger in this case, which can be understood as the charge in the $\mathrm{Ag}$ grating is more concentrated towards the $\mathrm{H}_{2} \mathrm{O} / \mathrm{Ag}$ surface.

\subsection{The effect of upper dielectric of the Ag grating}

In order to prevent the oxidation of Ag grating, a layer of $\mathrm{Al}_{2} \mathrm{O}_{3}$ was evaporated on the surface of $\mathrm{Ag}$ grating, forming a $\left(\mathrm{H}_{2} \mathrm{O}-\mathrm{Al}_{2} \mathrm{O}_{3}\right) / \mathrm{Ag} / \mathrm{NOA}$ heterostructure. By changing the thickness of $\mathrm{Al}_{2} \mathrm{O}_{3}$ thin layer, the influence of the change of refractive index of upper medium of $\mathrm{Ag}$ grating on the electromagnetic field of SERS substrate can be studied, as shown in Fig. 10.

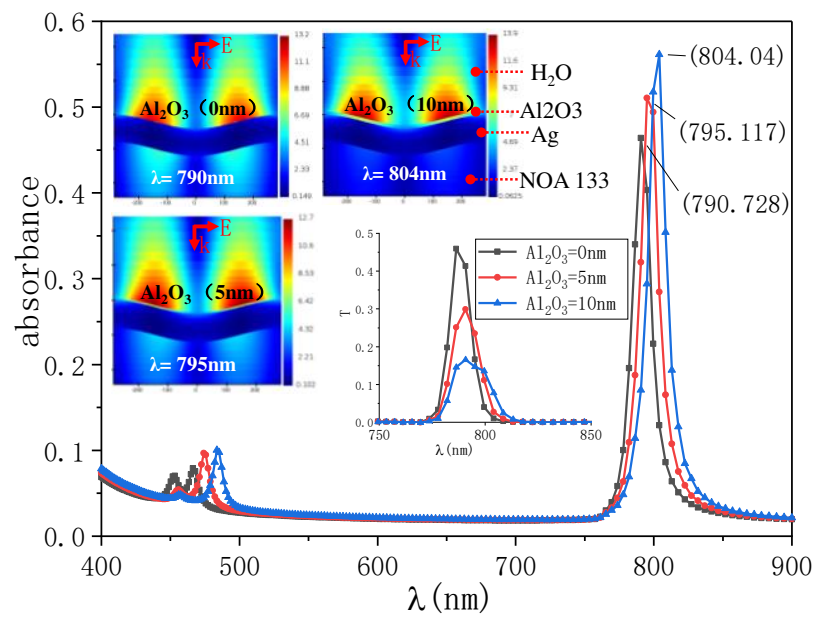

Fig. 10 SERS as a function of the thickness of $\mathrm{Al}_{2} \mathrm{O}_{3}$ with NOA133 and 80nm Ag

SPPs are coupled at the incident light of $786 \mathrm{~nm}$, where the $E_{x}(z)$ is an odd vector in Ag grating and the transmittance of SERS substrate is great, as shown in the transmittance curve in Figure 10. With the increasement of the thickness of $\mathrm{Al}_{2} \mathrm{O}_{3}$, the 
transmittance decreases, as the SPP on $\left(\mathrm{H}_{2} \mathrm{O}-\mathrm{Al}_{2} \mathrm{O}_{3}\right) / \mathrm{Ag}$ surface at $786 \mathrm{~nm}$ gradually weakens, resulting in the reduction of the odd modes of SPPs.

With the increase of $\mathrm{Al}_{2} \mathrm{O}_{3}$ thickness, the equivalent refractive index of $\left(\mathrm{H}_{2} \mathrm{O}-\mathrm{Al}_{2} \mathrm{O}_{3}\right)$ increases, leading to the red shift of SPP frequency of $\left(\mathrm{H}_{2} \mathrm{O}-\mathrm{Al}_{2} \mathrm{O}_{3}\right) / \mathrm{Ag}$ interface. As a result, the resonant frequency of the even modes redshifts and the coupling degree decreases gradually, as illustrated in the electric field distributions of even modes.

As can be seen from the transmittance curve and absorption curve in Figure 10, the transmittance of SERS substrate reaches the maximum in odd modes (786nm), while the absorbance reaches the maximum in even modes (such as $804 \mathrm{~nm}$ when $\mathrm{Al}_{2} \mathrm{O}_{3}$ is $10 \mathrm{~nm}$ ).

\section{Conclusion}

It has been shown that the interaction of gratingcoupled SPPs affects significantly the SERS intensity for Ag sinusoidal nanograting. This interaction is maximum at SPP resonance wavelength, when the refractive indexes of the superstrate is close to that of substrate. There are two modes of $E_{x}(z)$ in $\mathrm{Ag}$ grating, odd vector and even vector, when strong interaction of SPPs occurs. Generally speaking, the transmittance of SERS substrate is maximum in odd modes, while the absorbance is maximum in even modes.

With the decrease of Ag thickness, the interaction of SPPs is gradually enhanced, and the resonance frequency of odd modes is blue shifted, while that of even mode is red shifted. When the difference of refractive index between superstrate and substrate larger, the coupling of SPPs would decreases, and the transmittance of SERS substrate decreases correspondingly, while the EF of $\mathrm{H}_{2} \mathrm{O} / \mathrm{Ag}$ surface increases. As a consequence, the SERS is strongly dependent on the interaction of SPPs. The research may serve as a guideline for the design of metal/medium SERS substrate of multi-layer, as well as the substrate used in micro channel.

\section{Acknowledgments}

This work is supported by the National Defense Science Technology Project Fund (2004053).

\section{ORCID}

Zhaoyi Chen, https://orcid.org/0000-0002-7592-6284

\section{References}

[1] NAIR S, ESCOBEDO C, SABAT R G. Crossed Surface Relief Gratings as Nanoplasmonic Biosensors [J]. ACS sensors, 2017, 2(3): $379-85$.

[2] NAIR S, GOMEZ-CRUZ J, MANJARREZ-HERNANDEZ Á, et al. Selective Uropathogenic E. coli Detection Using Crossed SurfaceRelief Gratings [J/OL] 2018, 18(11):http://europepmc.org/abstract/MED/30373136

https://doi.org/10.3390/s18113634

https://europepmc.org/articles/PMC6263983

https://europepmc.org/articles/PMC6263983?pdf=render. $10.3390 / \mathrm{s} 18113634$

[3] GOMEZ-CRUZ J, NAIR S, MANJARREZ-HERNANDEZ A, et al Cost-effective flow-through nanohole array-based biosensing platform for the label-free detection of uropathogenic E. coli in real time [J]. Biosensors and Bioelectronics, 2018, 106: 105-10.

[4] CHUNG A J, HUH Y S, ERICKSON D. Large area flexible SERS active substrates using engineered nanostructures [J]. Nanoscale, 2011, 3(7): 2903-8

[5] DIES H, RAVEENDRAN J, ESCOBEDO C, et al. Rapid identification and quantification of illicit drugs on nanodendritic surface-enhanced Raman scattering substrates [J]. Sensors and Actuators B: Chemical, 2018, 257: 382-8.

[6] JIANG J, WANG X H, LI S, et al. Plasmonic nano-arrays for ultrasensitive bio-sensing [J]. Nanophotonics, 2018, 7(9): 1517-31.

[7] CHEN G, WANG Y, WANG H, et al. A highly sensitive microfluidics system for multiplexed surface-enhanced Raman scattering (SERS) detection based on Ag nanodot arrays [J]. RSC Advances, 2014, 4(97): 54434-40.

[8] PIOREK B D, LEE S J, MOSKOVITS M, et al. Free-Surface Microfluidics/Surface-Enhanced Raman Spectroscopy for RealTime Trace Vapor Detection of Explosives [J]. Analytical Chemistry, 
2012, 84(22): 9700-5.

[9] BARELLI M, GIORDANO M C, GUCCIARDI P G, et al. SelfOrganized Nanogratings for Large-Area Surface Plasmon Polariton Excitation and Surface-Enhanced Raman Spectroscopy Sensing [J]. Acs Applied Nano Materials, 2020, 3(9): 8784-93.

[10]CAO Y W C, JIN R C, MIRKIN C A. Nanoparticles with Raman spectroscopic fingerprints for DNA and RNA detection [J]. Science, 2002, 297(5586): 1536-40

[11]ZHANG C, MAN B Y, JIANG S Z, et al. SERS detection of lowconcentration adenosine by silver nanoparticles on silicon nanoporous pyramid arrays structure $[\mathrm{J}]$. Applied Surface Science, 2015, 347: 668-72.

[12]XU J, LI C, SI H, et al. 3D SERS substrate based on Au-Ag bi-metal nanoparticles/MoS2 hybrid with pyramid structure [J]. Optics Express, 2018, 26(17): 21546-57.

[13]LI C, YU J, XU S, et al. Constructing 3D and Flexible Plasmonic Structure for High-Performance SERS Application [J]. Advanced Materials Technologies, 2018, 3(11).

[14]MAP, LIANG F, DIAO Q, et al. Selective and sensitive SERS sensor for detection of $\mathrm{Hg} 2+$ in environmental water base on rhodaminebonded and amino group functionalized $\mathrm{SiO} 2$-coated $\mathrm{Au}-\mathrm{Ag}$ coreshell nanorods [J]. Rsc Advances, 2015, 5(41): 32168-74.

[15]LI J-F, ANEMA J R, WANDLOWSKI T, et al. Dielectric shell isolated and graphene shell isolated nanoparticle enhanced Raman spectroscopies and their applications [J]. Chem Soc Rev, 2015, 44(23): 8399-409

[16]LI C, XU S, YU J, et al. Local hot charge density regulation: Vibration-free pyroelectric nanogenerator for effectively enhancing catalysis and in-situ surface enhanced Raman scattering monitoring [J]. Nano Energy, 2021, 81.

[17]ZHAO X, LIU C, YU J, et al. Hydrophobic multiscale cavities for high-performance and self-cleaning surface-enhanced Raman spectroscopy (SERS) sensing [J]. Nanophotonics, 2020, 9(16): 476173.

[18]JAHN I J, ZUKOVSKAJA O, ZHENG X S, et al. Surface-enhanced Raman spectroscopy and microfluidic platforms: challenges, solutions and potential applications [J]. Analyst, 2017, 142(7): 102247.

[19]BURTSEV V, MILIUTINA E, ERZINA M, et al. Advanced Design of Microfluidic Chip Based on SPP-LSP Plasmonic Coupling for SERS Detection with High Sensitivity and Reliability [J]. Journal of Physical Chemistry C, 2019, 123(50): 30492-8.
[20]XIAO C, CHEN Z B, QIN M Z, et al. DERS substrate based on NERS-SERS interaction in integrated microfluidic detection [J] Appl Optics, 2018, 57(12): 3172-9

[21]KALACHYOVA Y, MARES D, JERABEK V, et al. Ultrasensitive and reproducible SERS platform of coupled Ag grating with multibranched Au nanoparticles [J]. Phys Chem Chem Phys, 2017, 19(22): 14761-9

[22]XIAO C, CHEN Z B, QIN M Z, et al. SPPs characteristics of $\mathrm{Ag} / \mathrm{SiO} 2$ sinusoidal nano-grating in SERS application $[\mathrm{J}]$. Optik, 2018, 168: 650-9.

[23]FAN M, ANDRADE G F S, BROLO A G. A review on recent advances in the applications of surface-enhanced Raman scattering in analytical chemistry [J]. Anal Chim Acta, 2020, 1097: 1-29.

[24]RAGHEB I, BRAIK M, LAU-TRUONG S, et al. Surface Enhanced Raman Scattering on Regular Arrays of Gold Nanostructures: Impact of Long-Range Interactions and the Surrounding Medium [J]. Nanomaterials, 2020, 10(11).

[25]NAIR S, GOMEZ-CRUZ J, ASCANIO G, et al. Cicada Wing Inspired Template-Stripped SERS Active 3D Metallic Nanostructures for the Detection of Toxic Substances [J]. Sensors, 2021, 21(5): 1699.

[26]XIAO C, CHEN Z B, QIN M Z, et al. SERS polarization-independent performance of two-dimensional sinusoidal silver grating $[\mathrm{J}]$. Appl Phys Lett, 2018, 113(17): 5.

[27]CHEN L L, LI J, LIU J, et al. A Ag-Au bimetallic nanograting surface plasmon resonance sensor based on a prism structure $[\mathrm{J}]$. Optics Communications, 2020, 461.

[28]ZHANG J X, ZHANG L D, XU W. Surface plasmon polaritons: physics and applications [J]. Journal of Physics D-Applied Physics, 2012, 45(11).

[29]IQBAL T, ASHFAQ Z, AFSHEEN S, et al. Surface-Enhanced Raman Scattering (SERS) on 1D Nano-gratings [J]. Plasmonics, 2020, 15(4): 1053-9.

[30]IQBAL T. Coupling Efficiency of Surface Plasmon Polaritons: Farand Near-Field Analyses [J]. Plasmonics, 2017, 12(1): 215-21.

[31]TALI S A S, ZHOU W. Multiresonant plasmonics with spatial mode overlap: overview and outlook [J]. Nanophotonics, 2019, 8(7): 1199. 225 .

[32]DE LOS SANTOS-SANCHEZ O. Probing intensity-field correlations of single-molecule surface-enhanced Raman-scattered light [J]. Frontiers of Physics, 2019, 14(6). 